\title{
HEINZ-SCHWARZ INEQUALITIES FOR HARMONIC MAPPINGS IN THE UNIT BALL
}

\section{David Kalaj}

University of Montenegro, Faculty of Natural Sciences and Mathematics Cetinjski put b.b. 81000 Podgorica, Montenegro; davidkalaj@gmail.com

\begin{abstract}
We first prove the following generalization of Schwarz lemma for harmonic mappings. If $u$ is a harmonic mapping of the unit ball onto itself then $\| u(x)-\left(1-\|x\|^{2}\right) /(1+$ $\left.\|x\|^{2}\right)^{n / 2} u(0) \| \leqslant U(|x| N)$. By using this result we obtain certain sharp estimate of the gradient of a harmonic mapping. Those two results extend some known result from harmonic mapping theory [1]. By using the Schwarz lemma for harmonic mappings we derive Heinz inequality on the boundary of the unit ball by providing a sharp constant $C_{n}$ in the inequality: $\left\|\partial_{r} u(r \eta)\right\|_{r=1} \geqslant C_{n}$, $\|\eta\|=1$, for every harmonic mapping of the unit ball into itself satisfying the condition $u(0)=0$, $\|u(\eta)\|=1$.
\end{abstract}

\section{Introduction}

Heinz in his classical paper [4] obtained the following result: If $u$ is a harmonic diffeomorphism of the unit disk $\mathbf{U}$ onto itself satisfying the condition $u(0)=0$, then

$$
\left|u_{x}(z)\right|^{2}+\left|u_{y}(z)\right|^{2} \geqslant \frac{2}{\pi^{2}}, \quad z \in \mathbf{U} .
$$

The proof uses the following representation of harmonic mappings in the unit disk

$$
u(z)=f(z)+\overline{g(z)}
$$

where $f$ and $g$ are holomorphic functions with $\left|g^{\prime}(z)\right|<\left|f^{\prime}(z)\right|$. It uses the maximum principle for holomorphic functions and the following sharp inequality

$$
\liminf _{r \rightarrow 1^{-}}\left|\frac{\partial u\left(r e^{i t}\right)}{\partial r}\right| \geqslant \frac{2}{\pi}
$$

proved by using the Schwarz lemma for harmonic functions. The aim of this paper is to generalize inequality (1.2) for several dimensional case.

If $u$ is a harmonic mapping of the unit ball onto itself, then we do not have any representation of $u$ as in (1.1).

It is well known that a harmonic function (and a mapping) $u \in L^{\infty}\left(B^{n}\right)$, where $B=B^{n}$ is the unit ball with the boundary $S=S^{n-1}$, has the following integral representation

$$
u(x)=\mathcal{P}[f](x)=\int_{S^{n-1}} P(x, \zeta) f(\zeta) d \sigma(\zeta)
$$

where

$$
P(x, \zeta)=\frac{1-\|x\|^{2}}{\|x-\zeta\|^{n}}, \quad \zeta \in S^{n-1}
$$

doi:10.5186/aasfm.2016.4126

2010 Mathematics Subject Classification: Primary 31A05; Secondary $42 B 30$.

Key words: Harmonic mappings, Heinz inequality, Schwarz inequality. 
is Poisson kernel and $\sigma$ is the unique normalized rotation invariant Borel measure on $S^{n-1}$ and $\|\cdot\|$ is the Euclidean norm.

We have the following Schwarz lemma for harmonic mappings on the unit ball $B^{n}$ (see e.g. [1]). If $u$ is a harmonic mapping of the unit ball into itself such that $u(0)=0$, then

$$
\|u(x)\| \leqslant U(r N)
$$

where $r=\|x\|, N=(0, \ldots, 0,1)$ and $U$ is a harmonic function of the unit ball into $[-1,1]$ defined by

$$
U(x)=\mathcal{P}\left[\chi_{S^{+}}-\chi_{S^{-}}\right](x),
$$

where $\chi$ is the indicator function and $S^{+}=\left\{x \in S: x_{n} \geqslant 0\right\}, S^{-}=\left\{x \in S: x_{n} \leqslant 0\right\}$. Note that, the standard harmonic Schwarz lemma is formulated for real functions only, but we can reduce the previous statement to the standard one by taking $v(x)=$ $\langle u(x), \eta\rangle$, for some $\|\eta\|=1$, where $\langle\cdot, \cdot\rangle$ is the Euclidean inner product. Indeed, we will prove a certain generalization of (1.4) without the a priory condition $u(0)=0$ (Theorem 2.1). For Schwarz lemma for the derivatives of harmonic mappings on the plane and space we refer to the papers $[6,7]$. It is worth to mention here a certain extension of (1.2) for the mappings which are solution of certain elliptic partial differential equations in the plane [2]. For certain boundary Schwarz lemma on the unit ball for holomorphic mappings in $\mathbf{C}^{n}$ we refer to the paper [9].

By using Hopf theorem it can be proved ([5]) that if $u$ is a harmonic mapping of the unit ball onto itself such that $u(0)=0$ and $\|u(\zeta)\|=1$, then

$$
\liminf _{r \rightarrow 1}\left\|\frac{\partial u}{\partial r}(r \zeta)\right\| \geqslant C_{n}
$$

where $C_{n}$ is a certain positive constant. Our goal is to find the largest constant $C_{n}$. This is done in Theorem 2.4 and Theorem 2.5.

\section{Preliminaries and main results}

First we prove the following extension and generalization of harmonic Schwarz lemma for $B^{n}, n \geqslant 3$. The case $n=2$ has been treated and proved by Pavlović [10, Theorem 3.6.1].

Theorem 2.1. If $u$ is a harmonic mapping of the unit ball onto itself, then

$$
\left\|u(x)-\frac{1-\|x\|^{2}}{\left(1+\|x\|^{2}\right)^{n / 2}} u(0)\right\| \leqslant U(\|x\| N) .
$$

Proof. Assume first that $x=r N$. We have that

$$
u(r N)=\int_{S^{n-1}} \frac{1-r^{2}}{\|\zeta-r N\|^{n}} f(\zeta) d \sigma(\zeta)
$$

and so

$$
u(r N)-\frac{1-r^{2}}{\left(1+r^{2}\right)^{n / 2}} u(0)=\int_{S^{n-1}}\left(\frac{1-r^{2}}{\|\zeta-r N\|^{n}}-\frac{1-r^{2}}{\left(1+r^{2}\right)^{n / 2}}\right) f(\zeta) d \sigma(\zeta) .
$$


Further we have

$$
\begin{aligned}
\left\|u(r N)-\frac{1-r^{2}}{\left(1+r^{2}\right)^{n / 2}} u(0)\right\| \leqslant & \int_{S^{n-1}}\left|\frac{1-r^{2}}{\|\zeta-r N\|^{n}}-\frac{1-r^{2}}{\left(1+r^{2}\right)^{n / 2}}\right| d \sigma(\zeta) \\
= & \int_{S^{+}}\left(\frac{1-r^{2}}{\|\zeta-r N\|^{n}}-\frac{1-r^{2}}{\left(1+r^{2}\right)^{n / 2}}\right) d \sigma(\zeta) \\
& +\int_{S^{-}}\left(\frac{1-r^{2}}{\left(1+r^{2}\right)^{n / 2}}-\frac{1-r^{2}}{\|\zeta-r N\|^{n}}\right) d \sigma(\zeta) .
\end{aligned}
$$

Thus

$$
\left\|u(r N)-\frac{1-r^{2}}{\left(1+r^{2}\right)^{n / 2}} u(0)\right\| \leqslant U(r N) .
$$

Now if $x$ is not on the ray $[0, N]$, we choose a unitary transformation $O$ such that $O(N)=x /|x|$. Then we make use of harmonic mapping $v(y)=u(O(y))$ for which we have $v(r N)=u(O(r N))=u(x)$. By making use of the previous proof we obtain (2.1).

In order to continue, recall the Khavinson question [7]. It deals with the sharp function $g(|x|)$ in the inequality $\|\nabla u(x)\| \leqslant g(|x|)\|u\|_{\infty}$, where $x$ is an arbitrary point of the unit ball. The variational problem of finding the coefficient $g(|x|)$ has been reduced in [8] to a solution of a minimization problem along a scalar parameter inside a double integral. By using Theorem 2.1, we obtain the following new proof of well-known inequality [11, p. 139, eq. (6)]. Observe that it is an extension of $[1$, Theorem 6.2.6].

Corollary 2.2. Under conditions of the previous theorem we have the following inequality

$$
\|\nabla u(x)\| \leqslant 2 \frac{\omega_{n-1}}{\omega_{n}} \frac{1}{1-\|x\|},
$$

where $\omega_{n}$ is the volume of $B^{n}$. The constant $2 \frac{\omega_{n-1}}{\omega_{n}}$ is sharp. However this inequality is not the sharp pointwise estimate, and thus it doesn't answer to the Khavinson question.

Proof. Let $x \in B^{n}$ and let $v(y)=u(x+(1-\|x\|) y)$. By applying (2.1) to $v$ we obtain

$$
\left\|u(x+(1-\|x\|) y)-\frac{1-\|y\|^{2}}{\left(1+\|y\|^{2}\right)^{n / 2}} u(x)\right\| \leqslant U(\|y\| N) .
$$

It follows that

$$
\left\|\frac{u(x+(1-\|x\|) y)-u(x)}{\|y\|}-\frac{\left(\frac{1-\|y\|^{2}}{\left(1+\|y\|^{2}\right)^{n / 2}}-1\right)}{\|y\|} u(x)\right\| \leqslant \frac{U(\|y\| N)}{\|y\|} .
$$

Since

we obtain that

$$
\lim _{\|y\| \rightarrow 0} \frac{\frac{1-\|y\|^{2}}{\left(1+\|y\|^{2}\right)^{n / 2}}-1}{\|y\|}=0
$$

$$
(1-\|x\|)\|\nabla u(x)\| \leqslant\left.\partial_{r} U(r N)\right|_{r=0}=2 \frac{\omega_{n-1}}{\omega_{n}} .
$$

2.1. Hypergeometric functions. In order to formulate and to prove our next results recall the basic definition of hypergeometric functions. For two positive 
integers $p$ and $q$ and vectors $a=\left(a_{1}, \ldots, a_{p}\right)$ and $b=\left(b_{1}, \ldots, b_{q}\right)$ we set

$$
{ }_{p} F_{q}[a ; b, x]=\sum_{k=0}^{\infty} \frac{\left(a_{1}\right)_{k} \cdots\left(a_{p}\right)_{k}}{\left(b_{1}\right)_{k} \cdots\left(b_{q}\right)_{k} \cdot k !} x^{k}
$$

where $(y)_{k}:=\frac{\Gamma(y+k)}{\Gamma(y)}=y(y+1) \ldots(y+k-1)$ is the Pochhammer symbol. The hypergeometric series converges at least for $|x|<1$. For basic properties and formulas concerning hypergeometric series we refer to the book [3]. The most important step in the proof of our main results, i.e., of Theorem 2.4 and Theorem 2.5 below, is the following lemma.

Lemma 2.3. The function $V(r)=\frac{\partial U(r N)}{\partial r}, 0 \leqslant r \leqslant 1$ is decreasing on the interval $[0,1]$ and we have

$$
V(r) \geqslant V(1)=C_{n}:=\frac{n !\left(1+n-(n-2){ }_{2} \mathrm{~F}_{1}\left[\frac{1}{2}, 1, \frac{3+n}{2},-1\right]\right)}{2^{3 n / 2} \Gamma\left[\frac{1+n}{2}\right] \Gamma\left[\frac{3+n}{2}\right]} .
$$

Proof. By using spherical coordinates $\eta=\left(\eta_{1}, \ldots, \eta_{n}\right)$ such that $\eta_{n}=\cos \theta$, where $\theta$ is the angle between the vector $x$ and $x_{n}$ axis, we obtain from (1.5) that

$$
U(r N)=\frac{\Gamma\left[\frac{n}{2}\right]}{\sqrt{\pi} \Gamma\left[\frac{n-1}{2}\right]} \int_{0}^{\pi} \frac{\left(1-r^{2}\right) \sin ^{n-2} \theta}{\left(1+r^{2}-2 r \cos \theta\right)^{n / 2}}\left(\chi_{S^{+}}(x)-\chi_{S^{-}}(x)\right) d \theta
$$

and so

$$
U(r N)=\frac{\Gamma\left[\frac{n}{2}\right]}{\sqrt{\pi} \Gamma\left[\frac{n-1}{2}\right]} \int_{0}^{\pi / 2}\left(\frac{\left(1-r^{2}\right) \sin ^{n-2} \theta}{\left(1+r^{2}-2 r \cos \theta\right)^{n / 2}}-\frac{\left(1-r^{2}\right) \cos ^{n-2} \theta}{\left(1+r^{2}+2 r \sin \theta\right)^{n / 2}}\right) d \theta
$$

or what can be written as

$$
U(r N)=\frac{\Gamma\left[\frac{n}{2}\right]}{\sqrt{\pi} \Gamma\left[\frac{n-1}{2}\right]} \int_{0}^{\pi / 2}\left(\frac{\left(1-r^{2}\right) \sin ^{n-2} \theta}{\left(1+r^{2}-2 r \cos \theta\right)^{n / 2}}-\frac{\left(1-r^{2}\right) \sin ^{n-2} \theta}{\left(1+r^{2}+2 r \cos \theta\right)^{n / 2}}\right) d \theta .
$$

Let $P=2 r /\left(1+r^{2}\right)$. Then

$$
\begin{aligned}
& \frac{\left(1-r^{2}\right) \sin ^{n-2} \theta}{\left(1+r^{2}-2 r \cos \theta\right)^{n / 2}}-\frac{\left(1-r^{2}\right) \sin ^{n-2} \theta}{\left(1+r^{2}+2 r \cos \theta\right)^{n / 2}} \\
& =\frac{\left(1-r^{2}\right)}{\left(1+r^{2}\right)^{n / 2}} \sum_{k=0}^{\infty}\left(\left(\begin{array}{c}
-n / 2 \\
k
\end{array}\right)\left((-1)^{k}-1\right) \cos ^{k} \theta \sin ^{n-2} \theta\right) P^{k}
\end{aligned}
$$

Since

we obtain

$$
\int_{0}^{\pi / 2} \cos ^{k} \theta \sin ^{n-2} \theta d \theta=\frac{\Gamma\left[\frac{1+k}{2}\right] \Gamma\left[\frac{1}{2}(-1+n)\right]}{2 \Gamma\left[\frac{k+n}{2}\right]}
$$

$$
U(r N)=\frac{\Gamma\left[\frac{n}{2}\right]}{\sqrt{\pi} \Gamma\left[\frac{n-1}{2}\right]} \frac{\left(1-r^{2}\right)}{\left(1+r^{2}\right)^{n / 2}} \sum_{k=0}^{\infty} \frac{\Gamma\left[\frac{1+k}{2}\right] \Gamma\left[\frac{n-1}{2}\right]}{2 \Gamma\left[\frac{k+n}{2}\right]}\left(\begin{array}{c}
-n / 2 \\
k
\end{array}\right)\left((-1)^{k}-1\right) P^{k} .
$$

Hence

where

$$
U(r N)=r\left(1-r^{2}\right)\left(1+r^{2}\right)^{-1-\frac{n}{2}} \frac{2 \Gamma\left[1+\frac{n}{2}\right]}{\sqrt{\pi} \Gamma\left[\frac{1+n}{2}\right]} G(r)
$$

$$
G(r)={ }_{3} \mathrm{~F}_{2}\left[1, \frac{2+n}{4}, \frac{4+n}{4} ; \frac{3}{2}, \frac{1+n}{2} ; \frac{4 r^{2}}{\left(1+r^{2}\right)^{2}}\right] \text {. }
$$


By [3, Eq. 3.1.8] for $a=\frac{n}{2}, b=\frac{1}{2}(-1+n), c=\frac{1}{2}$, we have that

$$
G(r)=\frac{\left(1+r^{2}\right)^{1+\frac{n}{2}}{ }_{4} F_{3}\left[\left\{\frac{n}{2}, \frac{1}{2}(-1+n), \frac{1}{2}, 1+\frac{n}{4}\right\},\left\{\frac{n}{4}, \frac{3}{2}, \frac{1}{2}+\frac{n}{2}\right\},-r^{2}\right]}{1-r^{2}} .
$$

So

$$
U(r N)=r \frac{2 \Gamma\left[1+\frac{n}{2}\right]}{\sqrt{\pi} \Gamma\left[\frac{1+n}{2}\right]}{ }_{4} F_{3}\left[\left\{\frac{n}{2}, \frac{1}{2}(-1+n), \frac{1}{2}, 1+\frac{n}{4}\right\},\left\{\frac{n}{4}, \frac{3}{2}, \frac{1}{2}+\frac{n}{2}\right\},-r^{2}\right],
$$

which can be written as

$$
U(r N)=\frac{2 \Gamma\left[1+\frac{n}{2}\right]}{\sqrt{\pi} \Gamma\left[\frac{1+n}{2}\right]} r+\sum_{k=1}^{\infty} \frac{2(-1)^{k}(4 k+n) \Gamma\left[k+\frac{n}{2}\right]}{(1+2 k)(-1+2 k+n) \sqrt{\pi} \Gamma[1+k] \Gamma\left[\frac{1}{2}(n-1)\right]} r^{2 k+1} .
$$

Thus

$$
\frac{\partial U(r N)}{\partial r}=\frac{2 \Gamma\left[1+\frac{n}{2}\right]}{\sqrt{\pi} \Gamma\left[\frac{1+n}{2}\right]}+\sum_{k=1}^{\infty} \frac{2(-1)^{k}(4 k+n) \Gamma\left[k+\frac{n}{2}\right]}{(-1+2 k+n) \sqrt{\pi} \Gamma[1+k] \Gamma\left[\frac{1}{2}(n-1)\right]} r^{2 k} .
$$

Since

$$
\begin{aligned}
& \frac{2(-1)^{k}(4 k+n) \Gamma\left[k+\frac{n}{2}\right]}{(-1+2 k+n) \sqrt{\pi} \Gamma[1+k] \Gamma\left[\frac{1}{2}(n-1)\right]} \\
& =\frac{(-1)^{k} 2^{n} \Gamma\left[1+\frac{n}{2}\right] \Gamma\left[k+\frac{n}{2}\right]}{\pi k ! \Gamma[n]}+\frac{2(-1)^{k}(-2+n) \Gamma\left[k+\frac{n}{2}\right]}{(-1+2 k+n) \sqrt{\pi} \Gamma[k] \Gamma\left[\frac{1+n}{2}\right]},
\end{aligned}
$$

we obtain that

$$
\frac{\partial U(r N)}{\partial r}=\frac{\Gamma\left[1+\frac{n}{2}\right]\left(\left(1+r^{2}\right)^{-n / 2}(1+n)-(n-2) r^{2}{ }_{2} F_{1}\left[\frac{1+n}{2}, \frac{2+n}{2}, \frac{3+n}{2},-r^{2}\right]\right)}{\sqrt{\pi} \Gamma\left[\frac{3+n}{2}\right]},
$$

which in view of the Kummer quadratic transformation, can be written in the form

$$
\frac{\partial U(r N)}{\partial r}=\frac{\Gamma\left[1+\frac{n}{2}\right]\left(1+r^{2}\right)^{-n / 2}\left(1+n-(n-2) r^{2}{ }_{2} F_{1}\left[\frac{1}{2}, 1, \frac{3+n}{2},-r^{2}\right]\right)}{\sqrt{\pi} \Gamma\left[\frac{3+n}{2}\right]} .
$$

The function

$$
y_{2} F_{1}[1 / 2,1,(3+n) / 2,-y]
$$

increases in $y$. Namely, its derivative is

$$
\begin{aligned}
{ }_{2} F_{1}[1 / 2,2,(3+n) / 2,-y] & =\sum_{m=0}^{\infty}(-1)^{m} a(m) y^{m} \\
& =\sum_{m=0}^{\infty} \frac{(-1)^{m}(1+m) \Gamma\left[\frac{1}{2}+m\right] \Gamma\left[\frac{3+n}{2}\right]}{\sqrt{\pi} \Gamma\left[\frac{3}{2}+m+\frac{n}{2}\right]} y^{m} .
\end{aligned}
$$

Then $a(m)>0$ and

$$
\frac{a(m)}{a(m+1)}=\frac{(1+m)(3+2 m+n)}{(2+m)(1+2 m)}>1,
$$

because $1+n+m n>0$, and so

$$
{ }_{2} F_{1}[1 / 2,2,(3+n) / 2,-y] \geqslant \sum_{m=0}^{\infty}(a(2 m)-a(2 m+1)) y^{2 m}>0 .
$$


The conclusion is that $\frac{\partial U(r N)}{\partial r}$ is decreasing. In particular,

$$
\frac{\partial U(r N)}{\partial r} \geqslant\left.\frac{\partial U(r N)}{\partial r}\right|_{r=1}
$$

For $r=1$ we have

$$
\frac{\partial U(r N)}{\partial r}=C_{n}=\frac{n !\left(1+n-(n-2){ }_{2} \mathrm{~F}_{1}\left[\frac{1}{2}, 1, \frac{3+n}{2},-1\right]\right)}{2^{3 n / 2} \Gamma\left[\frac{1+n}{2}\right] \Gamma\left[\frac{3+n}{2}\right]} .
$$

Theorem 2.4. If $u$ is a harmonic mapping of the unit ball into itself such that $u(0)=0$, then for $x \in B$ the following sharp inequality

$$
\frac{1-\|u(x)\|}{1-\|x\|} \geqslant C_{n}
$$

holds.

Proof. From Theorem 2.1 we have that $\|u(x)\| \leqslant U(r N)$ and so

$$
\frac{1-\|u(x)\|}{1-\|x\|} \geqslant \frac{1-|U(r N)|}{1-\|x\|} .
$$

Further there is $\rho \in(r, 1)$ such that

$$
\frac{1-U(r N)}{1-\|x\|}=\frac{\partial U(\rho N)}{\partial r}
$$

which in view of Lemma 2.3 is bigger that $C_{n}$. The proof is completed.

Theorem 2.5. (a) If $u$ is a harmonic mapping of the unit ball into itself such that $u(0)=0$, and for some $\|\zeta\|=1$ we have $\lim _{r \rightarrow 1}\|u(r \zeta)\|=1$, then

$$
\liminf _{r \rightarrow 1^{-}}\left\|\frac{\partial u}{\partial \mathbf{n}}(r \zeta)\right\| \geqslant C_{n} .
$$

(b) If $u$ is a proper harmonic mapping of the unit ball onto itself such that $u(0)=0$, then the following sharp inequality

$$
\liminf _{r \rightarrow 1^{-}}\left\|\frac{\partial u}{\partial \mathbf{n}}(r \zeta)\right\| \geqslant C_{n}, \quad\|\zeta\|=1
$$

holds. Here and in the sequel $\mathbf{n}$ is outward-pointing unit normal.

Proof. Prove (a). Then (b) follows from (a). Let $0<r<1$ and $x \in(r \zeta, \zeta)$. There is a $\rho \in(\|x\|, 1)$ such that

$$
\frac{1-\|u(x)\|}{1-r}=\left.\frac{\partial\|u(r \zeta)\|}{\partial r}\right|_{r=\rho} .
$$

On the other hand

$$
\left\|\frac{\partial u(r \zeta)}{\partial r}\right\| \geqslant \frac{\partial\|u(r \zeta)\|}{\partial r} .
$$

Letting $\|x\|=r \rightarrow 1$, in view of Thereom 2.4 and (2.4), we obtain that

$$
\liminf _{r \rightarrow 1}\left\|\frac{\partial u}{\partial \mathbf{n}}(r \zeta)\right\| \geqslant C_{n} .
$$


To show that the inequality (2.2) is sharp, let

$$
h_{m}(x)= \begin{cases}1-x / m, & \text { if } x \in(1 / m, 1] \\ (m-1) x, & \text { if }-1 / m \leqslant x \leqslant 1 / m \\ -1-x / m, & \text { if } x \in[-1,-1 / m)\end{cases}
$$

and define

$$
f_{m}\left(x_{1}, \ldots, x_{n-1}, x_{n}\right)=\frac{\sqrt{1-h_{m}\left(x_{n}\right)^{2}}}{\sqrt{1-x_{n}^{2}}}\left(x_{1}, \ldots, x_{n-1}, 0\right)+\left(0, \ldots, 0, h_{m}\left(x_{n}\right)\right) .
$$

Then $f_{m}$ is a homeomorphism of the unit sphere onto itself, such that

$$
\lim _{m \rightarrow \infty} f_{m}(x)=\left(0, \ldots, 0, \chi_{S^{+}}(x)-\chi_{S^{-}}(x)\right) \text {. }
$$

Further, $u_{m}(x)=\mathcal{P}\left[f_{m}\right](x)$ is a harmonic mapping of the unit ball onto itself such that $\lim _{\|x\| \rightarrow 1}\left\|u_{m}(x)\right\|=1$. Thus $u_{m}$ is proper. Moreover, $u_{m}(0)=0$ and $\lim _{m \rightarrow \infty} u_{m}(x)=$ $(0, \ldots, 0, U(x))$. This implies the fact that the constant $C_{n}$ is sharp.

By taking $v(x)=u(x)-\frac{1-\|x\|^{2}}{\left(1+\|x\|^{2}\right)^{n / 2}} u(0)$ and following the proof of Theorem 2.5, in view of Theorem 2.1 we obtain the following theorem.

Theorem 2.6. (a) If $u$ is a harmonic mapping of the unit ball into itself, and for some $\|\zeta\|=1$ we have $\lim _{r \rightarrow 1}\|u(r \zeta)\|=1$, then

$$
\liminf _{r \rightarrow 1^{-}}\left\|\frac{\partial u}{\partial \mathbf{n}}(r \zeta)+\frac{u(0)}{2^{n / 2-1}}\right\| \geqslant C_{n} .
$$

(b) If $u$ is a proper harmonic mapping of the unit ball onto itself, then the sharp inequality (2.5) holds for $\|\zeta\|=1$.

In particular, when $n=2$, the inequality (2.5) reads as

$$
\liminf _{r \rightarrow 1^{-}}\left\|\frac{\partial u}{\partial \mathbf{n}}(r \zeta)+u(0)\right\| \geqslant \frac{2}{\pi} .
$$

Remark 2.7. The following table shows first few constants $C_{n}$ and related functions.

\begin{tabular}{|c|c|c|c|}
\hline$n$ & $U(r N)$ & $\partial_{r} U(r N)$ & $C_{n}$ \\
\hline 2 & $\frac{4 \arctan (r)}{\pi}$ & $\frac{4}{\pi\left(1+r^{2}\right)}$, & $\frac{2}{\pi}$ \\
\hline 3 & $\frac{-1+r^{2}+\sqrt{1+r^{2}}}{r \sqrt{1+r^{2}}}$ & $\frac{1-\sqrt{1+r^{2}}-r^{2}\left(-3+\sqrt{1+r^{2}}\right)}{r^{2}\left(1+r^{2}\right)^{3 / 2}}$ & $\sqrt{2}-1$ \\
\hline 4 & $\frac{2 r\left(-1+r^{2}\right)+2\left(1+r^{2}\right)^{2} \arctan r}{\pi r^{2}\left(1+r^{2}\right)}$ & $\frac{4\left(r+3 r^{3}-\left(1+r^{2}\right)^{2} \arctan r\right)}{\pi r^{3}\left(1+r^{2}\right)^{2}}$ & $\frac{4-\pi}{\pi}$ \\
\hline
\end{tabular}

\section{References}

[1] Axler, S., P. Bourdon, and W. RAmey: Harmonic function theory. - Springer-Verlag, New York, 1992.

[2] Chen, S., and M. Vuorinen: Some properties of a class of elliptic partial differential operators. - J. Math. Anal. Appl. 431:2, 2015, 1124-1137.

[3] Gasper, G., and M. Rahman: Basic hypergeometric series. - Cambridge Univ. Press, 2004.

[4] Heinz, E.: On one-to-one harmonic mappings. - Pacific J. Math. 9, 1959, 101-105.

[5] Kalaj, D., and M. Mateljević: Harmonic quasiconformal self-mappings and Möbius transformations of the unit ball. - Pacific J. Math. 247:2, 2010, 389-406. 
[6] Kalaj, D., and M. Vuorinen: On harmonic functions and the Schwarz lemma. - Proc. Amer. Math. Soc. 140:1, 2012, 161-165.

[7] Khavinson, D.: An extremal problem for harmonic functions in the ball. - Canad. Math. Bull. $35: 2,1992,218-220$.

[8] Kresin, G., and V. MAZ'YA: Sharp pointwise estimates for directional derivatives of harmonic functions in a multidimensional ball. - J. Math. Sci. (N. Y.) 169:2, 2010, 167-187.

[9] Liu, T., J. WAng, and X. TAng: Schwarz lemma at the boundary of the unit ball in $\mathbf{C}^{n}$ and its applications. - J. Geom. Anal. 25:3, 2015, 1890-1914.

[10] PaVlović, M.: Introduction to function spaces on the disk. - Matematički institut SANU, Belgrade, 2004.

[11] Protter, M. H., and H.F. Weinberger: Maximum principles in differential equations. Prentice-Hall Partial Differential Equations Series, Prentice-Hall, Englewood Cliffs, N.J., 1967.

Received 3 July 2015 • Revised received 18 September 2015 • Accepted 2 October 2015 